\title{
Structures, Fields and Methods of Identification of Nonlinear Static Systems in the Conditions of Uncertainty
}

\author{
Nikolay N. Karabutov \\ Moscow State Institute of Radio Engineering, Electronics and Automation, Russia \\ E-mail:kn22@yandex.ru \\ Received March 8, 2010; revised September 2, 2010; accepted September 13, 2010
}

\begin{abstract}
The field of structures on set of secants is offered and methods of its construction for various classes of one-valued nonlinearities of static systems are considered. The analysis of structural properties of system is fulfilled on specially generated set of data. Representation on which modification it is possible to judge to nonlinear structure of static systems is introduced. It is shown, that structures of nonlinear static systems have a special $V$-point. The adaptive algorithm of an estimation of structure of nonlinearity on a class polynomial function is offered.
\end{abstract}

Keywords: Structure, Identification, Algorithm, Field of Structures, Nonlinear System

\section{Introduction}

Statistical methods are applied to a solution of a problem of structural identification basically, based on correlative and an analysis of variance [1,2]. Various approaches and methods are applied to identification of nonlinear systems. In [1-3] the statistical methods based on the correlation and dispersive analysis are used. In identification systems genetic algorithms [4-6] are widely applied. For synthesis of models neural networks [7-11] and their combination with various methods of approximation $[12,13]$ also are used. Genetic and neural network algorithms allow to find structure of model of nonlinear systems on the set of approximating functions. In a number of works the aprioristic information [14-16] and the subsequent approximation on the set class of functions [17-19] is used. Thus the choice of a class of functions is not always proved. The carried out analysis shows, that despite set of the publications, any formalized procedures of an estimation of structure it was offered not as the considered subject domain is very difficult for describing in existing language of mathematical concepts, objects and decision-making. The problem of structural identification even more becomes complicated for static systems as for them it is complicated to open the existing correlations, expressing through the integrated indicator - a system exit. Here a priori data can render the big help. In the conditions of a priori uncertainty informational synthesis of system can give the additional information only.

Attempt to estimate area to which should belong required nonlinear structure is natural. For nonlinear dynamic systems the concept of a sector condition [20], to which should belong investigated nonlinearity has been introduced. This condition is applied only at a stage of synthesis of dynamic systems. In identification systems to formalize such condition till now it was not possible. For the first time such attempt has been undertaken in $[21,22]$ for dynamic systems where on the basis of analysis of results of measurements the set which contains the information on nonlinearity has been generated, and also the method of structural identification is offered. Properties of nonlinear dynamic systems can be estimated on a phase or observable informational portrait (OIP). For static systems of such universal graphic representation does not exist. The problem even more becomes complicated that in the majority of the real systems described by static models, the informational set generated on the basis of results of measurements, has irregular character.

More low the approach to construction of a field of structures on a class of secants for nonlinear static systems which is development and generalization of the outcomes received in $[21,22]$ is offered. The concept of a sector condition is introduced and algorithms of a decision making about structure of nonlinear system are offered. The structure on which it is possible to make a solution on nonlinearity of system is offered. 


\section{Field of Structures on Class $F_{m}$}

The plant is considered

$$
\begin{gathered}
y_{n}=A^{T} U_{n}+f(U, n)+\xi_{n} \\
f(U, n) \in F_{m}=\left\{\begin{array}{c}
u_{i, n} \in R, u_{i, n} \in U \mid f: R \times J_{N} \rightarrow R, \\
f\left(U_{n}\right)=\sum_{i}^{m} \sum_{j}^{p} \alpha_{i j} u_{i, n} u_{j, n}, \\
i \neq j, 1 \leq(m, p)<k
\end{array}\right\},
\end{gathered}
$$

where $U_{n} \in R^{k}, y_{n} \in R$ is an input and a output, $A \in \Omega_{A} \subset R^{k}$ is a vector of parameters, belong limited, but a priori unknown area $\Omega_{A}, \alpha_{i j}$ is some numbers, $n \in J_{N}$ is discrete time, $\xi_{n} \in R$ is a disturbance on an output.

For (1) the informational set is known

$$
\mathrm{I}_{o}=\mathrm{I}_{o}(y, U)=\left\{y_{n}, U_{n}, n \in J_{N}=[0, N]\right\}
$$

and mapping $\Gamma_{o}: U \times y \quad \forall n \in J_{N}$ corresponding to it. Mapping $\Gamma_{o}$ describes an observable informational portrait. Restriction OIP $\left.\Gamma_{o}^{u_{i}} \subset \Gamma_{o}\right|_{u_{i} \in U} \forall i=\overline{1, k}$ is defined and for everyone $\Gamma_{o}^{u_{i}}$ secant

$\bar{\gamma}\left(y, u_{i}\right)=a_{0, i}+a_{1, i} u_{i, n}$, where $a_{0, i}, a_{1, i}$ is some real numbers is constructed.

Secants $\bar{\gamma}\left(y, u_{l} u_{j}\right)$ for $u_{l} u_{j} \in F_{m}$ are constructed. Let introduce the set of secants

$$
\bar{\Gamma}(U, y)=\left\{\bar{\gamma}\left(y, u_{i}\right), \bar{\gamma}\left(y, u_{l}, u_{j}\right), i=\overline{i, k},(l, j) \geq 1\right\}
$$

set on $\mathrm{I}_{o}$.

Definition 1. A field of structures $S_{S}$ of system (1) on class $F_{m}$ we will name a collection of mappings

$\bar{\gamma}\left(y, u_{i}\right) \subset u_{i} \times y \quad \forall i=\overline{1, k}, \quad \bar{\gamma}\left(y, u_{l} u_{j}\right) \subset u_{l} u_{j} \times y$

$\exists(l, j) \geq 1$ on Euclidean plane $\mathrm{E}$

$$
S_{S}\left(F_{m}\right)=\left\{\bar{\gamma}\left(y, u_{i}\right) \forall i=\overline{1, k}, \bar{\gamma}\left(y, u_{l} u_{j}\right), l \neq j,(l, j) \geq 1\right\} .
$$

It is necessary on the basis of analysis $\Gamma_{o}, \mathrm{I}_{o}$ to construct a field of structures $S_{S}$ for system (1) on set $\bar{\Gamma}(U, y)$.

As mapping $\Gamma_{o}$ at $k \geq 2$ does not give in to obvious interpretation, that, we will be limited to its restrictions $\left.\Gamma_{o}^{u_{i}} \subset \Gamma_{o}\right|_{u_{i} \in U} \quad \forall i=\overline{1, k}$ constructed on plane $\left(u_{i}, y\right)$. As a result we will receive some final set of mappings $\Gamma_{o}^{u_{i}}, \Gamma_{o}^{u_{l} u_{j}}$, which represents a field of structures $S_{S}$ of system (1) on plane E.

As secants with various ranges of definition the area of definition of field $S_{S}\left(F_{m}\right)$ on plane $\mathrm{E}$ will be equal $\bigcup \operatorname{dom}\left(u_{i}\right)$ are considered, and area of values $S_{S}\left(F_{m}\right)$ ${ }_{\text {will coincide with a area of values of mapping } \Gamma_{o}}$

$$
\operatorname{rng}\left(S_{S}\right)=\operatorname{rng}\left(\Gamma_{o}\right)=\operatorname{rng}(y) .
$$

Let construct mappings $\Gamma_{o}^{u_{i}}, \Gamma_{o}^{u_{l} u_{j}}$ on plane E. We will add to them elements of set $\bar{\Gamma}(U, y)$. Further we will be limited only to the analysis of properties $\bar{\gamma}\left(y, u_{i}\right)$, $\bar{\gamma}\left(y, u_{l} u_{j}\right)$.

The field of structures allows to simplify the analysis of structure of system (1) and to estimate as degree of linearity (nonlinearity) of system through available set of secants, and influence of elements of vector $U \in R^{k}$ on shaping of a nonlinear part of system $f(U, n)$. Corresponding results are more low give. The problem of localization of function $f(U, n)$ on the basis of available set of experimental data $I_{o}$ is not less important. In conditions uncertainty the solution of the given problem is connected with overcoming of some problems. In work it is shown, that one of the approaches, allowing to localize required area, it is based on sector construction on set of secants. The quantity indicator (coherence coefficient), allowing on the basis of properties of secants to decision making about belong of nonlinearity to sector is introduced.

Theorem 1 [21]. The system (1) with nonlinearity $f(U, n) \in F_{m}$ has a linear field of structures $S_{S}\left(F_{m}\right)$.

Let consider sector $S_{i j} \subset S_{S}\left(F_{m}\right)$ limited to secants $\bar{\gamma}\left(y, u_{i}\right), \bar{\gamma}\left(y, u_{j}\right)$. These secants have angular coefficients $a_{1, i}, a_{1, j}$. We consider, that $a_{1, i}<a_{1, j}$. If it will appear, that in this sector almost secant $\bar{\gamma}\left(y, u_{l}, u_{j}\right)$ probably lays, i.e. Its angular coefficients belongs to interval $\left(a_{1, i}, a_{1, j}\right)$ component $u_{i} u_{j} \in F \quad$ can be included in structure of model for system (1). The substantiation of this statement will be more low given. The analysis of all candidates a priori entering into function $f(U, n)$ in (1) similarly realize.

With each element $\bar{\gamma}_{q} \in \bar{\Gamma}(U, y), q=\overline{1, \# \bar{\Gamma}(U, y)}$, where $\# \bar{\Gamma}(U, y)$ is a cardinal number $\bar{\Gamma}(U, y)$, we will associate local structures of static system. We will designate through $r_{\vartheta_{q} y}$ coefficient of mutual correlation between $\vartheta_{q}$ and $y$.

Definition 2. We will say, that variable $u_{l} u_{j}$ is coherent with $y$ or has relationship with $y$, if secant $\bar{\gamma}\left(y, u_{l}, u_{j}\right) \in S_{i j} \subset S_{S}\left(F_{m}\right)$ almost sure. In this case sector $S_{i j}$ we will name area of a coherence of variable $u_{l} u_{j}$.

For an estimation of closeness of relation $u_{l} u_{j}$ with $y$ we will introduce coherence coefficient

$$
Q_{i j}=r_{u_{i} y} / r_{u_{j} y} .
$$

Let notice, that $Q_{i j} \leq 1$ and, basically, should be close to $r_{u_{i} u_{j} y}$. If the given condition is not fulfilled, it speaks about incoherence to considered component $u_{i} u_{j}$.

Theorem 2 [21]. If the coefficient of coherence $Q_{i j}$ is equal $Q_{i j}=\delta_{i j}$, where $\delta_{i j} \geq \min \left(r_{u_{i} y}, r_{u_{j} y}\right)$, and $\delta_{i j}=r_{u_{i} u_{j} y}$ secant $\bar{\gamma}_{i j}=\bar{\gamma}\left(y, u_{i} u_{j}\right)$ belongs to sector $S_{i j}$.

The stated approach remains fair also for a class of 
nonlinearities $\mathcal{F}_{d}$

$$
f(U, n) \in F_{d}=\left\{\begin{array}{c}
u_{i} \in R, u_{i} \in U, U \in R^{k} \mid \\
f: R \times J_{N} \rightarrow R, \\
f\left(U_{n}\right)=\sum_{l}^{m} \sum_{j}^{p} \alpha_{l j} u_{l, n}^{d_{l}} u_{j, n}^{d_{j}}, \\
l \neq j, 1 \leq(m, p)<k
\end{array}\right\},
$$

where $d_{j}, d_{l}$ its are some numbers.

So, the method of secants allows to carry out the analysis of structural properties of static system on the basis of construction of a field of structures. The basic virtue of the given approach is the possibility of representation of structure of system on set of linear functions (secants). Analysis $S_{S}$ allows to select those elements from class $F_{m}$, for which

$$
\left(\min \left(r_{u_{i} y}, r_{u_{j} y}\right) \leq Q_{i j}\right) \&\left(u_{i} u_{j} \in S_{i j}\right) .
$$

The field of structures can be constructed for a wide class of static systems. On the basis of $S_{S}\left(F_{m}\right)$ it is possible to make an inference about structure of static system in the conditions of uncertainty and by that essentially to narrow a class of research models.

The field of structures can be under construction on set $\mathrm{I}_{e}$ [21-23], i.e. the set, the received ambassador of elimination linear making of $y_{n}$. It is easy to prove correspondence of fields of structures on sets $\mathrm{I}_{o}$ and $\mathrm{I}_{e}$ only for those $f(U, n) \in F_{m}$ for which for factor of coherence $Q_{i j}$ the Theorem 2 is fulfilled.

The example of construction of a field of structures is reduced in $[22,23]$.

\section{Field of Structures on Class $F_{s}$}

Let's spread the approach offered above to a class of nonlinearities

$$
\begin{gathered}
f(U, n) \in \mathcal{F}_{s}=\left\{u_{i} \in R, u_{i} \in U, U \in R^{k} \mid f: R \times J_{N} \rightarrow R,\right. \\
\left.f\left(u_{i}, d\right)=\alpha_{i} u_{i}{ }^{d}, d<\infty, \alpha_{i}<\infty\right\}_{s} .
\end{gathered}
$$

Let show, how for class $F_{s}$ to receive coherence area, i.e. sector to which belong nonlinearity $f(U, n)=u_{i, n}^{d_{i}}$, $d_{i}<\infty$. The further account is based on a straightening method [21,22]. The field of secants is under construction on set $\left\{k_{e, u_{i}, n}, e_{n}\right\}$, where $e_{n} \in \mathrm{I}_{e}$ is an error of forecasting of an output signal of system $y_{n}$ by means of model $\hat{y}_{k_{c}}=\hat{a} s_{n}$, where $s_{n}=I^{T} U_{n}, I \in R^{k}$ is an unit vector, $k_{e, u_{i}}$ is coefficient structural properties [21]

$$
k_{e, u_{i}, n}=k_{s}\left(e, u_{i}, n\right)=e_{n} / u_{i, n} .
$$

Let consider restriction $\Gamma_{e} \subset\left\{k_{e, u_{i}, n}\right\} \times\left\{e_{n}\right\}$ and its contractions $\Gamma_{e}^{k_{e, u_{i}}} \forall i=\overline{1, k}$. For everyone $\Gamma_{e}^{k_{e, u_{i}}}$ we will construct a secant

$$
\bar{\gamma}\left(e, k_{e, u_{i}}\right)=\bar{\gamma}_{i}=a_{e, 0}^{i}+a_{e, 1}^{i} k_{e, u_{i}} .
$$

Secants $\bar{\gamma}\left(e, k_{e, u_{j}, d_{j}}\right)$ for mapping $\Gamma_{e}^{k_{e, u_{j}, d_{j}}} \forall u_{j}^{d_{j}} \in \mathcal{F}_{s}$ $(j \geq 1)$ are set

$$
\bar{\gamma}\left(e, k_{e, u_{i}, d_{i}}\right)=\bar{\gamma}_{i, d_{i}}=a_{e, 0, d_{i}}+a_{e, 1, d_{i}} k_{e, u_{i}, d_{i}},
$$

where $a_{e, 0, d_{i}}, a_{e, 1, d_{i}}$ is some numbers,

$$
k_{e, u_{j}, d_{j}, n}=k_{s}\left(e, u_{j}, d_{j}, n\right)=e_{n} / u_{j, n}^{d_{j}}, d_{j}<\infty .
$$

Let's introduce set of secants

$$
\bar{\Gamma}(K, e)=\left\{\bar{\Gamma}\left(K_{e}, e\right), \bar{\Gamma}\left(K_{e, d}, e\right)\right\} \quad \forall e_{n} \in \mathrm{I}_{e},
$$

where $\bar{\Gamma}\left(K_{e}, e\right)=\left\{\bar{\gamma}\left(e, k_{e, u_{i}}\right), i=\overline{1, k}\right\}$,

$K_{e}=\left[k_{e, u_{1}}, \ldots, k_{e, u_{k}}\right]^{T}, k_{e, u_{i}, d_{i}} \in K_{e, d}, K_{e, d} \in R^{q}, q \leq k$, $\bar{\Gamma}\left(K_{e, d}, e\right)=\left\{\bar{\gamma}\left(e, k_{e, u_{i}, d_{j}}\right), j \geq 1\right\}$.

Statement 1. Any secant $\bar{\gamma}\left(e, k_{e, u_{i}}\right) \in \bar{\Gamma}\left(K_{e}, e\right)$ at $j=i$ limits element $\bar{\gamma}\left(e, k_{e, u_{j}, d_{j}}\right) \in \bar{\Gamma}\left(K_{e, d}, e\right)$ from below.

Upper bound of sector $S_{d_{i}}$ to which should belong secant $\bar{\gamma}\left(e, k_{e, u_{i}, d_{i}}\right)$, on the basis of set $\bar{\Gamma}\left(K_{e}, e\right)$ to define it is not possible. Therefore to the received field of structures we will add secant $\bar{\gamma}\left(e, k_{e, s_{i}, d_{i}}\right)$, where $s_{i} \in R, s_{i}=I^{T}\left(U / u_{i}\right)$. As $k_{e, s}$ represents a transmission coefficient between $s_{n} \in R$ and $e_{n}$, and local coefficient structural properties $k_{e, u_{i}}$ are stationary the same property will possess and $k_{e, s_{i}}$. Hence, the coefficient of determination $\bar{\gamma}\left(e, k_{e, s_{i}, d_{i}}\right)$ will be not less $r_{k_{u_{i}} e}^{2}$ and $r_{k_{e, j} d_{i} e}^{2}$ at least for $0<d_{i}<1$. Thus, $\bar{\gamma}\left(e, k_{e, s_{i}, d_{i}}\right)$ it is possible to use as an upper bound of area of coherence $S_{d_{i}}$. It is fair, as transformation $u_{i, n}^{d_{i}}$ conducts to a modification only the statistical moments of signal $u_{j}$, but not its structures. In the supposition, that $0<d_{i}<1$, we receive reduction of the two first statistical moments that leads to a raise of factor of determination $\bar{\gamma}\left(e, k_{e, s_{i}, d_{i}}\right)$.

The field of structures for system (1) on class $F_{s}$ looks like

$$
S_{S}\left(F_{s}\right)=\left\{\bar{\Gamma}(K, e),\left\{\bar{\gamma}\left(e, k_{e, s_{i}, d_{i}}\right)\right\}\right\},
$$

where $d_{i} \in(0 ; 1), \quad 1 \leq i \leq k$.

In structure of model of system (1) we will include those functions $u_{i}^{d_{i}} \in F_{s}$ for which condition $r_{k_{i} e}^{2} \leq r_{k_{e, u_{i}, d_{i} e} e}^{2}$ is satisfied. The evaluation of coefficient of a coherence (2) in this case leads to an inadequate estimation of connection between $e$ and $u_{i}^{d_{i}}$.

Let's reduce algorithm of decision-making relative to structure of model of system (1) on class $F_{s}$.

1) We build secants $\bar{\gamma}\left(e, k_{e, u_{i}}\right)$ on plane $\left(k_{e, u_{i}}, e\right)$ for $\forall \Gamma_{e}^{k_{e}, u_{i}}$.

2) We define coefficient of determination $r_{k_{e, u_{i} e}}^{2}$ for 
$\forall \bar{\gamma}\left(e, k_{e, u_{i}}\right)$.

3) If $r_{k_{e, u_{i}} e}^{2} \geq \delta_{r}$, where $\delta_{r}>0$ is some set quantity the system is linear on variable $u_{i} \in U$.

4) If condition $r_{k_{e, u_{i}} e}^{2} \geq \delta_{r}$ is not fulfilled, is find $\bar{\gamma}\left(e, k_{e, s_{i}, d_{i}}\right)$ and defined $r_{k_{e, u_{i}, d_{i} e}}^{2}$. At $r_{k_{e, u_{i}, d_{i} e}}^{2}>\delta_{r}$ element $u_{i, n}^{d_{i}} \in F_{s}$ is included in model structure.

Remark. Condition $r_{k_{e_{u}} e}^{2} \geq \delta_{r}$ is an indication of linearity of system on variable $u_{i} \in U$.

$\Gamma_{e}$ for class $F_{s}$ it is possible to present mapping also in space $\left(K_{e},\left|e_{n}\right|\right)$. Thus result of synthesis will not.

On Figure 1 the field of structures $S_{S}\left(F_{s}\right)$ for plant (1) with vector $A=\left[\begin{array}{llll}0.8 & 1.4 & 2\end{array}\right]^{T}$, function $f(U, n)=0,5 u_{2, n}^{0,3}$ and limited noise $\xi_{n}$ is reduced ary. For secants $\Gamma_{e}$ following values of coefficients of determination are received: $r_{k_{e, u_{1}} e}^{2}=0.97, r_{k_{e, u_{2}} e}^{2}=0.91$, $r_{k_{\beta_{1}, u_{3}} e}^{2}=0.98 . \delta_{r}=0.94$. Comparison of received values $r^{2, u_{3} e}$ with $\delta_{r}$ has allowed to exclude $F_{s}$ elements $u_{1}^{d_{1}}$, $u_{3}^{d_{3}}$ from set. For $u_{2}^{d_{2}}$ on the basis of a straightening method value $d_{2}=0.3$, and $r_{k_{e, u_{1}, 0,3^{e}}^{2}}^{2}=0.99$ is received. On Figure 1 the area of coherence $S_{d_{2}}$ for variable $u_{2}^{d_{2}}$ is shown.

So, the method of construction of a field of structures $S_{S}$ on sets $F_{m}$ and $F_{s}$ for nonlinear system (1) is offered. It is based on the analysis of an informational portrait of system and in many respects depends on an kind of class $F_{m}$. Very often (class $F_{s}$ ) portrait $\Gamma_{e}$ is under construction in the space received by transformation of initial variables of system of identification. It speaks, in particular, complexity of a problem of structural identification for which solution nontrivial approaches and methods should be attracted. In particular, on class $F_{s}$ for decision-making on structure of model of system (1) field of structures $S_{S}\left(F_{s}\right)$ is under construction on set of coefficients structural properties systems as it is a measure of linearity of system and allows to establish degree of a deviation from this indicator of performances of system.

\section{Field of Structures on Class $F_{f}$}

Let consider plant (1) with nonlinearity

$$
\begin{gathered}
f(U, n) \in \mathcal{F}_{f}=\left\{u_{i} \in R, u_{i} \in U \mid f: R \times J_{N} \rightarrow R,\right. \\
\left.f\left(u_{i, n}\right)=\sum_{\alpha} c_{\alpha} u_{i, n}^{\alpha}, \alpha<\infty, i \geq 1\right\},
\end{gathered}
$$

where $c_{\alpha}$ is some numbers.

Let on the basis of $\mathrm{I}_{o}$ set $\mathrm{I}_{e}=\left\{U_{n}, e_{n}, n \in J_{N}\right\}$, where $e_{n}=\hat{y}_{n}-y_{n}, \hat{y}_{n}=\hat{a} s_{n}$ is generated.

It is necessary for class $F_{f}$ on the basis of handling of set $\mathrm{I}_{e}$ to construct a field of structures for system (1). For a problem solution we will take advantage of the approach stated in Section 2. We take variable $u_{i}$ for

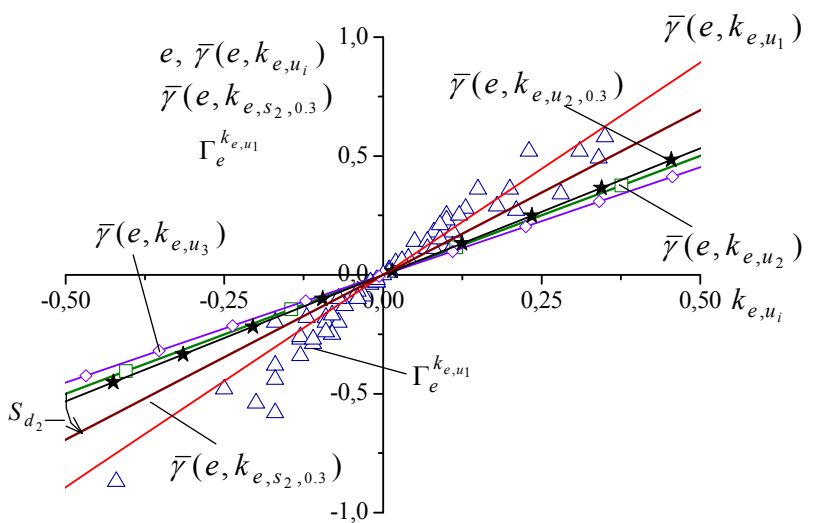

Figure 1. A field of structures of system (1) with $f(U, n) \in F_{s}$ ary.

which condition $r_{u_{i} e} \geq \delta_{e}$, where $\delta_{e}>0$ is some set magnitude is satisfied. We will construct for $u_{i}$ mapping $\Gamma_{e} \subset\left\{k_{e, u_{i}, n}\right\} \times\left\{e_{n}\right\}$ and a secant

$$
\bar{\gamma}\left(e, k_{e, u_{i}}\right)=\bar{\gamma}_{i}=a_{e, 0}^{i}+a_{e, 1}^{i} k_{e, u_{i}} .
$$

Let consider function $u_{i}^{\alpha}$ and for everyone $\alpha \in J_{\alpha}=[1 ; \bar{\alpha}]$ we will define coefficient structural properties $k_{e, u_{i}, \alpha}$, where $J_{\alpha}$ is some segment of the real numbers which choice will be given more low.

For everyone $\alpha \in J_{\alpha}$ we will construct secant $\bar{\gamma}\left(e, k_{e, u_{i}, \alpha}\right)$ (3) for mapping $\Gamma_{e}^{k_{e, u_{i}, \alpha}}$. $\alpha$ we will change until for coefficient of determination of secant $\bar{\gamma}\left(e, k_{e, u_{i}, \alpha}\right)$ the condition will be satisfied

$$
r_{k_{e, u_{i}, \alpha^{e}}^{2}}^{2} \geq \tilde{\delta}_{e}
$$

where $\tilde{\delta}_{e}=\tilde{\delta}_{e}\left(\delta_{e}\right)>0$.

Let introduce set of secants

$$
\begin{gathered}
\bar{\Gamma}\left(k_{e, u_{i}, \alpha}, e\right)=\left\{\bar{\gamma}\left(e, k_{e, u_{i}, \alpha}\right) \forall \alpha \in J_{\alpha}\right. \\
\text { such, that } \left.r_{k_{e, u_{i}, \alpha}}^{2} \geq \tilde{\delta}_{e}\right\}
\end{gathered}
$$

Upper bound $\bar{\alpha}$ of interval $J_{\alpha}$ is defined from a condition of violation (6).

Definition 3. We will name set $\bar{\Gamma}\left(k_{e, u_{i}, \alpha}, e\right)$ a field of structures $S_{S}\left(F_{f}, \bar{\Gamma}\left(k_{e, u_{i}, \alpha}, e\right)\right)$ of the system (1), covering nonlinearity $f(U, n)$ on class $F_{f}$.

The field of structures for system (1) on class $F_{f}$ looks like

$$
S_{S}\left(F_{f}, \bar{\Gamma}\left(k_{e, u_{i}, \alpha}, e\right)\right)=\left\{\bar{\Gamma}\left(k_{e, u_{i}, \alpha}, e\right), \alpha \in J_{\alpha}, i \geq 1\right\} .
$$

Let consider secant $\bar{\gamma}\left(e, k_{e, u_{i}, \alpha}\right)$ with indicator $\alpha=\bar{\alpha}$ and sector $S_{\alpha}=\left(\bar{\gamma}\left(e, k_{e, u_{i}, 1}\right), \bar{\gamma}\left(e, k_{e, u_{i}, \bar{\alpha}}\right)\right)$. We will define coefficient of coherence $Q_{\alpha}$ on class $F_{f}$

$$
Q_{\alpha}=r_{k_{e, u_{i}, \bar{\alpha}} e} / r_{k_{e, u_{i}, l^{e}}} \text {. }
$$

On $Q_{\alpha}$ we will judge membership $f\left(u_{i, n}\right)$ to sector 
$S_{\alpha}$.

Let consider a choice of magnitude $\tilde{\delta}_{e}$ in (6), and, therefore, and $\bar{\alpha}$. For everyone $u_{i, n}^{\alpha}$ we will calculate coefficient of correlation $r_{u_{\dot{\alpha}}^{\alpha}}$. We will define parameter $\bar{\alpha} \in J_{\alpha}$ from a condition of violation of an inequality

$$
\left(\max _{\alpha} r_{u_{i}^{\alpha} e} \geq \delta_{e}\right) \rightarrow \bar{\alpha}
$$

On the basis of $\bar{\alpha}$ we will set magnitude $\tilde{\delta}_{e}$ in (6) and we will find $Q_{\alpha}$.

Theorem 3. Let for system (1) the field of structures $S_{S}\left(F_{f}\right)$ is constructed. If condition $r_{f\left(u_{i}\right), e} \geq Q_{\alpha}$ nonlinearity $f\left(u_{i, n}\right) \in F_{f}$ is coherent with signal $y_{n}$ is satisfied and it can be included in model structure.

On set $\left\{u_{i, n}^{\alpha}\right\}$ the field of structures be of the form

$$
S_{S}\left(F_{f}, \bar{\gamma}\left(e, u_{i}^{\alpha}\right)\right)=\left\{\bar{\gamma}\left(e, u_{i}^{\alpha}\right) \forall \alpha \leq \bar{\alpha}\right\} .
$$

Unlike classes $F, F_{s}$, field $S_{S}\left(F_{f}\right)$ allows to estimate structure of nonlinearity $f(U, n)$ on final set of approximating functions $u_{i}^{\alpha}$ with index $\alpha \in J_{\alpha}$. In some cases by an amount of members of an approximating polynomial it is possible to set structure of function $f(U, n)$.

The example of a field of structures $S_{S}\left(F_{f}, \bar{\Gamma}\left(k_{e, u_{i}, \alpha}, e\right)\right)$ for system (1) with nonlinearity $f\left(u_{1}\right)=\sin \left(u_{1}\right)$ and a vector of parameters $A=\left[\begin{array}{llll}1.3 & 1.6 & 1.7\end{array}\right]^{T}$ is shown on Figure 2.

In Figure 2 following symbols are used: a straight line with a rhomb is a secant with $\alpha=1$; a straight line with quadrate is a secant with $\alpha=2$; a straight line with a triangle is a secant with $\alpha=2.5$; a straight line with a circle is a secant with $\alpha=3$. Here the example of modification $e_{n}$ for case $\alpha=1$ is shown. Maximum value in (7) is reached at $\bar{\alpha}=2.5$. For this value $\tilde{\delta}_{e}=0.81$. $Q_{\alpha}=0.81 . r_{\sin \left(u_{i}\right), e}=0.83$ and, therefore, $f\left(u_{1, n}\right)$ is coherent with $y_{n}$. Let's notice, that at $\alpha=(0 ; 2)$

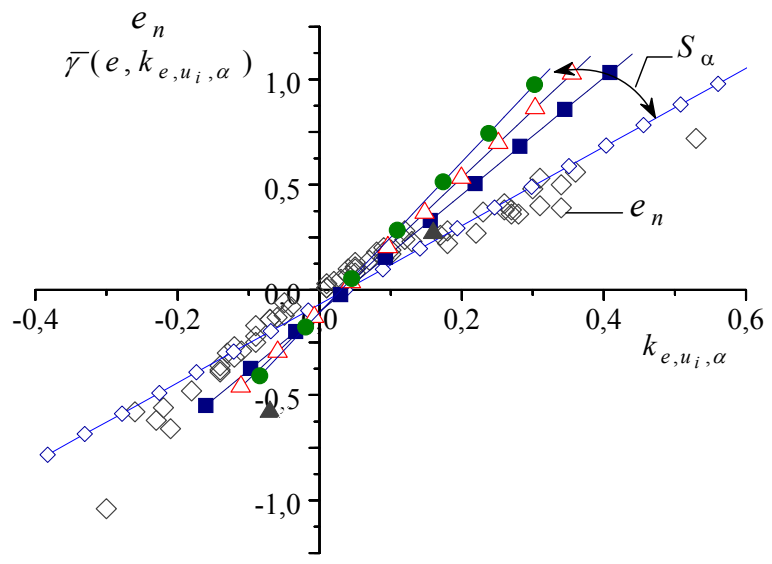

Figure 2. A field of structures of system (1) on class $F_{f}$ with nonlinearity $f\left(u_{1}\right)=\sin \left(u_{1}\right)$. $f\left(u_{1}\right) \cong \cos \left(u_{1}\right)$, and at $\alpha=(1 ; 3) \quad f\left(u_{1}\right) \cong \sin \left(u_{1}\right)$, and adequacy of model with $f\left(u_{1}\right)=f_{c}=\cos \left(u_{1}\right)$ on set $\mathrm{I}_{e}$ above, than with $f\left(u_{1}\right)=f_{s}=\sin \left(u_{1}\right)$. For decision-making concerning structure $f\left(u_{1}\right)$ in the field $S_{S}\left(F_{f}, \bar{\Gamma}\left(k_{e, u_{i}, \alpha}, e\right)\right)$ we fulfil check of candidates $f_{c}, f_{s}$ on set $\mathrm{I}_{o}$. Results of an estimation of adequacy speak about necessity for model to use element $f_{s} \in F_{f}$.

Remark. If to build a field of structures $S_{S}\left(F_{f}\right)$ on set $\mathrm{I}_{o}$ owing to low coefficients of correlation for decision-making it is necessary to pass in space of coefficients structural properties. Here it is completely applicable the approach stated above.

The offered approach allows to estimate nonlinearity structure through variable $e_{n}$. It is connected with that, the problem of estimation $f(U, n) \in F_{f}$ on set $\mathrm{I}_{e}$ demands performance double approximation, that in a considered case is not realized. Further the method of identification of function $f(U, n) \in F_{f}$ on a basis on the adaptive approach is offered.

\section{Adaptive Procedure of an Estimation of Function $\boldsymbol{f}(\boldsymbol{U}, \boldsymbol{n})$ on Class $F_{f}$}

Let it is known variable $u_{i} \in U$ and maximum degree $\alpha=\bar{\alpha}$ in (5). We will designate

$$
X_{n}=\left[\begin{array}{llll}
u_{i, n} & u_{i, n}^{2} & \ldots & u_{i, n}^{\bar{\alpha}}
\end{array}\right]^{T} \in R^{p}, C=\left[\begin{array}{llll}
c_{1} & c_{2} & \ldots & c_{p}
\end{array}\right]^{T} \in R^{p} .
$$

Desired dependence looks like

$$
e_{n}=\mu f\left(u_{i, n}\right)=\mu C^{T} X_{n},
$$

where $\mu \in R$.

For estimation $\mu, C$ it is applicable adaptive model

$$
\hat{e}_{n}=\hat{\mu}_{n-1} \hat{f}\left(u_{i, n}\right)=\hat{\mu}_{n-1} \hat{C}_{n-1}^{T} X_{n}
$$

where $\hat{\mu}_{n}, \hat{C}_{n}$ is adjusted parameters.

Let designate an error of prediction $e_{n}$ by means of model (9) through $\varepsilon_{n}=\hat{e}_{n}-e_{n}$. We will introduce misalignment $v_{n}=\hat{f}\left(u_{i, n}\right)-\hat{f}\left(u_{i, n}\right)$.

Algorithms of adaptation $\hat{\mu}_{n}, \hat{C}_{n}$ we search from a condition

$$
\min _{\hat{\mu}_{n}} \varepsilon_{n}^{2} \rightarrow \hat{\mu}^{o}, \min _{\hat{C}_{n}} v_{n}^{2} \rightarrow \hat{C}^{o},
$$

From (10) it is received

$$
\begin{aligned}
& \hat{C}_{n}=\hat{C}_{n-1}-\gamma_{C} \varepsilon_{n} X_{n}, \\
& \hat{\mu}_{n}=\hat{\mu}_{n-1}-\gamma_{\mu} v_{n} \hat{C}_{n-1}^{T} X_{n},
\end{aligned}
$$

where $\gamma_{C}>0, \gamma_{\mu}>0$ is the parameters ensuring convergence of algorithms.

As function $f\left(u_{i, n}\right)$ is unknown, we will use estimation $f\left(u_{i, n}\right)=e_{n} / \hat{\mu}_{n-1}$. 
Let note the Equations (11), (12) are rather misalignment model parameters

$$
\begin{gathered}
\delta C_{n}=\delta C_{n-1}-\gamma_{C} v_{n} X_{n}, \\
\delta \mu_{n}=\delta \mu_{n-1}-\gamma_{\mu} \varepsilon_{n} \hat{C}_{n-1}^{T} X_{n},
\end{gathered}
$$

where $\delta C_{n}=\hat{C}_{n}-C_{n}, \delta \mu_{n}=\hat{\mu}_{n}-\mu_{n}$.

Theorem 4. Let $|\mu| \leq \alpha_{\mu},\left\|X_{n}\right\|<\infty$, and also exists such $0<\alpha_{\varepsilon} \leq \alpha_{\mu}^{-1}$, that $\varepsilon_{n} v_{n} \leq \alpha_{\varepsilon} \varepsilon_{n}^{2}$. Then algorithms (13), (14) converge, if

$$
\begin{gathered}
0<\gamma_{C} \leq \frac{2}{\left\|X_{n}\right\|^{2}}, \\
0<\gamma_{\mu} \leq 2\left(1-\alpha_{\varepsilon} \alpha_{\mu}\right) / \beta,
\end{gathered}
$$

where $\left|\hat{C}_{n-1}^{T} X_{n}\right| \leq \beta \quad \forall n \geq 0,\|\cdot\|$ is Euclidean norm. If $e_{n}$ contains uncertainty $\zeta_{n}$ limited on level $\left|\zeta_{n}\right| \leq \vartheta$ Algorithms (13), (14) will converge if it is fulfilled (15) and

$$
\begin{gathered}
\vartheta \leq\left(1-\alpha_{\varepsilon} \alpha_{\mu}\right) \max _{n}\left|\varepsilon_{n}\right|, \\
0<\gamma_{\mu} \leq \frac{2\left[\left(1-\alpha_{\varepsilon} \alpha_{\mu}\right)-\rho\right]}{\beta},
\end{gathered}
$$

where $\rho<1$.

Theorem 5. Algorithms (13), (14) do not possess an asymptotic stability.

So, Algorithms (13), (14) do not allow to receive asymptotically an estimation of parameters of system (8). Here uncertainty in which algorithms are applied affects.

The example of work of adaptive system of identification of nonlinear system (1) with $f\left(u_{1}\right)=\sin \left(u_{1}\right)$ and $A=\left[\begin{array}{lll}1.3 & 1.6 & 1.7\end{array}\right]^{T}$ is shown on Figures 3, 4. Vector $X_{n}=\left[\begin{array}{ll}u_{i, n} & u_{i, n}^{2}\end{array}\right]^{T}$. The Figure 3 reflects process of tuning of parameters of model (9). On Figure 4 the informational portrait reflecting results of adequacy of an estimation of function $f\left(u_{1}\right)$ is shown. The determination coefficient was equaled 0.99 , that follows from position of secant $\bar{\gamma}(\hat{f}, f)$. The expectation and average quadratic deviation for $f\left(u_{1}\right)$ and $\hat{f}\left(u_{1}\right)$ are accordingly equal:

$$
\begin{gathered}
\bar{f}\left(u_{1}\right)=0.78, \quad \overline{\hat{f}}\left(u_{1}\right)=0.767, \sigma\left(f\left(u_{1}\right)\right)=0.26, \\
\sigma\left(\hat{f}\left(u_{1}\right)\right)=0.28
\end{gathered}
$$

So, the mode of construction of a field of structures for nonlinear static system (1) on the basis of the analysis of set $\mathrm{I}_{e}$ is offered. The sector condition is introduced and the mode of its construction for various classes of nonlinearities is offered. The analysis of structural properties is fulfilled in space of secants of an observable informational portrait of system or its virtual analogues.

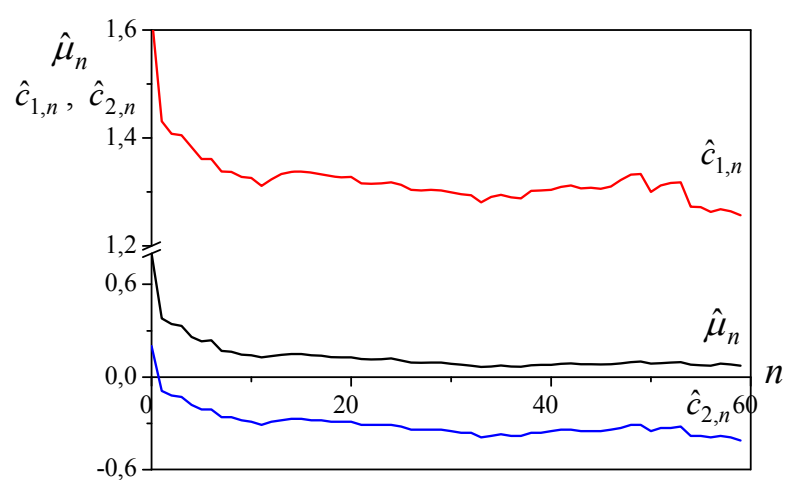

Figure 3. Tuning of parameters of model (9).

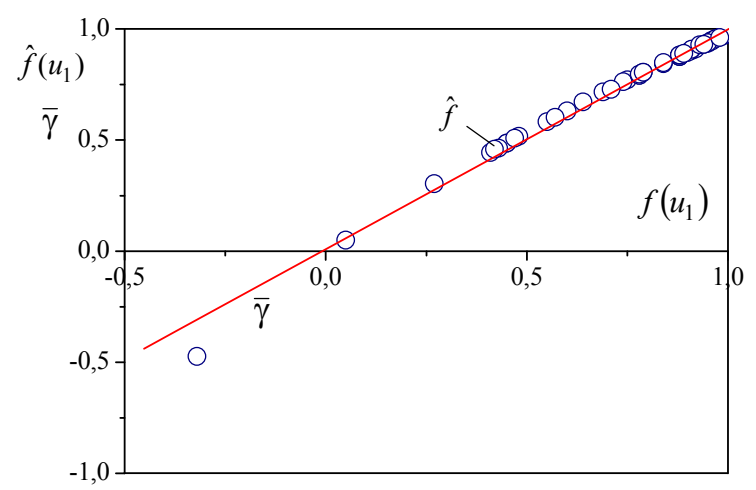

Figure 4. An estimation of adequacy of model (9) on plane $(f, \hat{f})$.

Algorithms and methods of identification of nonlinear component system are developed for various classes of nonlinearities.

\section{Structures of Nonlinear Static Systems}

The approaches stated above allow to make for a various class of nonlinearities a solution on structure of model of system. Field $S_{S}$ gives conception about system structure on a vector space of secants. Naturally there is a problem on search of mapping for nonlinear static system which would allow to make imprisonment before trail about its nonlinear properties. Complexities of introduction such mapping were considered above. Despite it, the informational structures, allowing to receive conception about nonlinearity of static system are more low offered.

Let consider informational set

$$
\mathrm{I}_{e}=\mathrm{I}_{e}(e, U)=\left\{e_{n}, U_{n}, n \in J_{N}=[0, N]\right\} .
$$

Appropriate $\mathrm{I}_{e}$ informational portrait $\Gamma_{e}:\left\{U_{n}\right\} \times\left\{e_{n}\right\}$ owing to an operation of perturbation $\xi_{n} \in R$ has irregular character and the solution does not allow to make on nonlinear properties of system (1). The told confirms 
Figure 5 on which the informational portrait for the plant considered in Section 3 is shown.

Let consider set

$$
\mathrm{I}_{k}=\mathrm{I}_{k}(e, k)=\left\{e_{n}, k_{n}, n \in J_{N}=[0, N]\right\},
$$

where $k_{n} \in R$ is factor structural properties systems (1) on class $F_{r} \quad(r=m, s, f)$ for $u_{i, n} \in U_{n}$.

Let order values $k_{n}$ on increase on set $J_{N}$, that is we will construct a variation number series. As a result we will receive set $\left\{k_{q}^{v}\right\}$, where $q \in J_{N}^{v}=[0, N]$. To everyone $k_{q}^{v}$ there corresponds value $e_{q}^{v}$. Hence

$$
\mathrm{I}_{k}^{v}=\mathrm{I}_{k}^{v}(e, k)=\left\{e_{q}^{v}, k_{q}^{v}, q \in J_{N}^{v}=[0, N]\right\} .
$$

On $\mathrm{I}_{k}^{v}$ we will construct mapping $\Gamma_{e}^{v}:\left\{k_{q}^{v}\right\} \times\left\{e_{q}^{v}\right\}$ to which on Euclidean plane $\left(k_{q}^{v}, e_{q}^{v}\right)$ there corresponds some structure $S_{k e}^{v}$. We assume, that function $e_{q}^{v}$ is simple, univalent and intersects axis $k_{q}^{v}$ in some point, that is $S_{k e}^{v}$ has a singular point. $e_{n}$ represents an error of prediction of an exit of system (1) on the basis of linear static model with input $s_{n}=I^{T} U_{n} . k_{q}^{v}$ also is function $e_{n}$. Therefore mapping $\Gamma_{e}^{v}$ will represent some function, tending growth. It is fair for any class $F_{r}$. It is known [21], that for linear static systems the coefficient structural properties is an estimation of parameter of model for $u_{i, n} \in U_{n}$. For nonlinear systems it represents the function varying in some limited range. Starting with $\mathrm{I}_{k}^{v}$ it is concluded, that process of modification $k_{q}^{v}$ at magnification $q$ has monotone character. The same character will be carried also by function $e_{q}^{v}$ but as $e_{q}^{v}$ depends from $f(U, n)$ and $\xi_{n}$ its modification will differ from the linear. For an estimation of degree of nonlinearity we will construct secant $\bar{\gamma}\left(e_{q}^{v}, k_{q}^{v}\right)$. Hence, unlike $\Gamma_{e}$ mapping $\Gamma_{e}^{v}$ is structurally informative. So, fairly

Statement 2. For system (1) with $f(U, n) \in F_{r}$ $(r=m, s, f)$ on set $\mathrm{I}_{k}^{v}$ there is mapping

$\Gamma_{e}^{v}:\left\{k_{q}^{v}\right\} \times\left\{e_{q}^{v}\right\}$ to which on Euclidean plane $\left(k_{q}^{v}, e_{q}^{v}\right)$ there corresponds the informational structure $S_{k, e}^{v}$, allowing to analyze nonlinear properties of system.

Unlike $\mathrm{I}_{e}$ on set $\mathrm{I}_{k}^{v}$ structure $S_{k, e}^{v}$ has more ordered character. It is necessary to notice, that $S_{k, e}^{v}$ it is applicable also for mapping of linear static systems. For $\forall f(U, n) \in F_{r} \quad(r=m, s, f)$ structure $S_{k e}^{v}$ contains a singular point, who unlike dynamic systems is not characteristic performance of a stability of system (1). She serves as confirmation of a monotonicity of the curve described by mapping $\Gamma_{e}^{v}$. If to take advantage Lyapunov's $\lambda$ of characteristic indexes they, as one would expect, state the estimation of index $\lambda$ close to zero.

Let state a method, allowing to define type of a singular point for the given class of systems. We will introduce functions

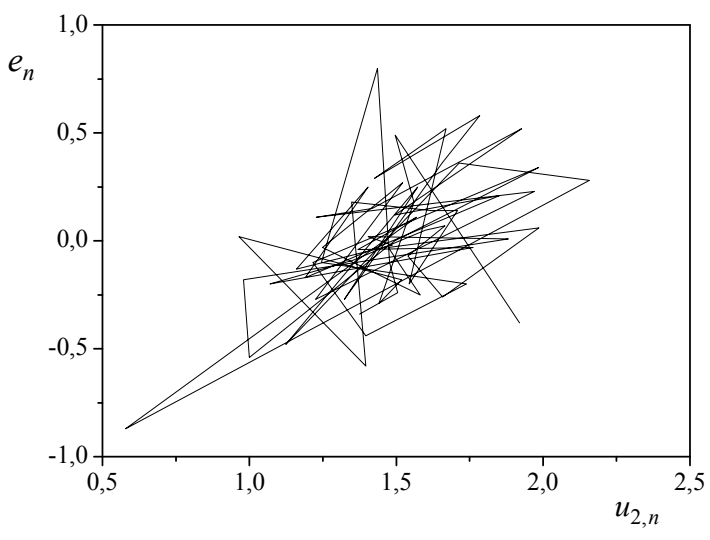

Figure 5. Projection of informational portrait $\Gamma_{e}$ to plane $\left(u_{2}, e\right)$.

$$
v_{k, q}=\ln \left|k_{q}^{v}\right|, v_{e, q}=\ln \left|e_{q}^{v}\right|
$$

Let find such value $q \in J_{N}^{v}$, for which

$$
\min _{q} v_{k, n} \rightarrow q_{o}, \min _{q} v_{e, n} \rightarrow q_{o} .
$$

Knowing $q_{o}$, from (24) we define values $k_{q, o}^{v}, e_{q, o}^{v}$, and, therefore, and singular point $M\left(k_{q, o}^{v}, e_{q, o}^{v}\right)$. We name $M\left(k_{q, o}^{v}, e_{q, o}^{v}\right) \quad V$-point. This title follows from the following. Let's construct on plane $\left(v_{k, q}, v_{e, q}\right)$ a portrait of system (1) to which there corresponds structure $S \mathcal{L}^{v}$. As show simulation data, for system (1) with $f(U, n) \in F_{r}$ it will look like, shown on Figure 6.

On Figure 6 the structure of nonlinear system (1) in spaces $\left(\Upsilon_{k, q}, \Upsilon_{e, q}\right),\left(\mathcal{K}_{k, q}, \mathcal{E}_{e, q}\right)$ is presented. From Figure 6 we see, that $\left(\mathscr{Y}_{k, q}, \mathscr{\Upsilon}_{e, q}\right)$ structure $S \mathcal{L}_{k}^{v}$ contains $V$-point in space. In her the rate of motion of points of a trajectory with magnification $q$ comes nearer to zero, reaching the minimum value at $q=q_{o}$. Then $\forall q>q_{o}$ the rate of motion of a point again increases. Such behaviour of a trajectory speaks properties of the logarithm. So, fairly

Statement 3. In space $\left(\Upsilon_{k, q}, \Upsilon_{e, q}\right)$ structure $S \mathcal{L}_{k, e}^{v}$ of system (1) has a special $V$-point.

Structure $S_{k, e}^{v}$ in space $\left(\mathcal{K}_{k, q}, \mathcal{E}_{e, q}\right)$ allows to estimate nonlinear properties of system. From Figure 6 it is see, that $S_{k, e}^{v}$ has nonlinear character and reflects a state system (1) ${ }^{k, e}$ with $f(\cdot) \in F_{s}, f(\cdot)=0.5 u_{2, n}^{0,3}$. Representation $S^{v}$ is more informative, than a portrait on Figure 5. For decision-making on that, how much the change of trajectory $S_{k, e}^{v}$ differs from linear, it is possible to take advantage of methods of secants or straightening.

Representation $S_{k,}^{v}$ for some class of nonlinearities allows to make a solution on structure function $f$.

Theorem 6. Let the coefficient structural properties $k_{q}^{v}$ is defined on segment $J_{k}^{v}=\left[\underline{k}_{q}^{v}, \bar{k}_{q}^{v}\right]$, where $\underline{k}_{q}^{v}<\infty$, $\bar{k}_{q}^{v}<\infty, q \in J_{N}^{v}, f(U, n) \in F_{s}$. If exists such $\stackrel{q}{d}_{*} \neq 0$, 


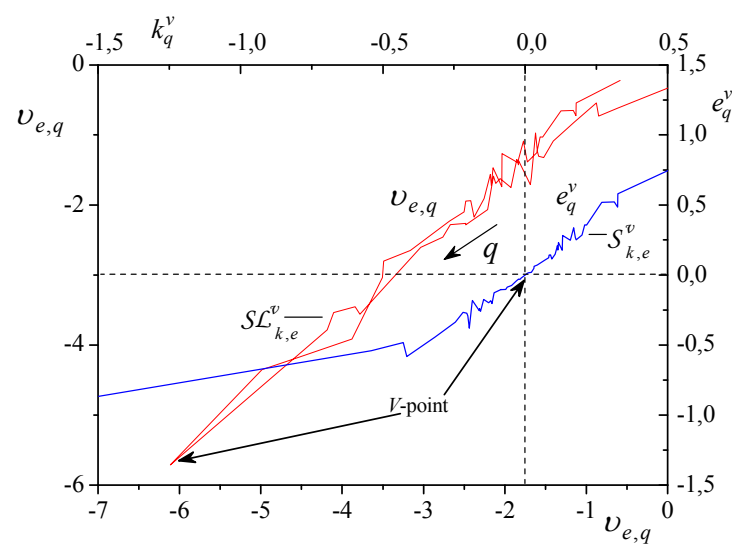

Figure 6. Informational structures $S \mathcal{L}_{k, e}^{v} S_{k, e}^{v}$ of system (1).

that

$$
\left|\underline{k}_{q}^{v} / \bar{k}_{q}^{v}\right| \mid \in O(1)
$$

where $O(1)$ is some neighborhood $1 f\left(u_{i}, d_{*}\right)$ defines structure of nonlinearity of system (1) on class $F_{s}$.

Remark. The Theorem 6 gives the new formulation of a method of the straightening stated in Section 3. In her the criterion of linearity directly is used.

The Theorem 6 is applicable only to so-called controllable models [21].

Let consider structure $S_{k, e}^{v}$ of system (1) on class $F_{f}$. In this case the theorem 6 is not applicable, so function $f(U, n)$ explicitly does not contain the controllable parameter. For a choice of an amount of members in an approximating Polynomial (5) it is possible to take advantage of the approach stated in Section 4. As informational set it is necessary to use $\left\{k_{e, u_{i}, \alpha, q}^{v}, e_{q}^{v}\right\}, q \in J_{N}^{v}$.

Let consider nonlinearity $f\left(u_{i}, u_{j}\right) \in F_{m},\left(u_{i}, u_{j}\right) \in U$. Let generate sets $\mathrm{I}_{k}^{v}\left(e, k_{e, u_{i}}\right), \mathrm{I}_{k}^{v}\left(e, k_{e, u_{j}}\right), \mathrm{I}_{k}^{v}\left(e, k_{e, u_{i} u_{j}}\right)$ to which there correspond structures $S_{i}=S_{k, e}^{v}\left(\mathrm{I}_{u_{i}}^{v}\right)$ $S_{j}=S_{k, e}^{v}\left(\mathrm{I}_{u_{j}}^{v}\right), S_{i j}=S_{k, e}^{v}\left(\mathrm{I}_{u_{i} u_{j}}^{v}\right)$. For each structure secant $\bar{\gamma}_{\tau}$ is received $(\tau=i, j, i j), \bar{\gamma}_{\tau}=\bar{\gamma}\left(e^{v}, k_{e, u_{\tau}}^{v}\right) . \bar{\gamma}_{\tau}$ it is characterized by parameters $a_{0, i}, a_{1, i}$.

Theorem 7. Let on Euclidean plane $\left(k_{e, u_{i}, q}^{v}, e_{q}^{v}\right)$ structures $S_{i}, S_{j}, S_{i j}$, sector $S=\left(S_{i}, S_{j}\right)$ to which belong secants $\bar{\gamma}_{i}, \bar{\gamma}_{j}$, and $a_{1, i}<a_{1, j}$ are constructed. Then $S_{i j}=S$, if for almost $\forall q \in J_{N}^{v}$

$$
\pi_{i}\left(e_{q, i}^{v}-a_{o, i}\right) \leq e_{q, i j}^{v}-a_{o, i j} \leq \pi_{j}\left(e_{q, j}^{v}-a_{o, j}\right),
$$

where

$$
\pi_{i}=\frac{\min _{q} e_{q}}{\max _{g} e_{q}} \frac{\min _{q} u_{q, i}^{v}}{\max _{g}\left(u_{q, i} u_{q, j}\right)^{v}},
$$

$$
\pi_{j}=\frac{\max _{q} e_{q}}{\min _{g} e_{q}} \frac{\max _{q} u_{q, i}^{v}}{\min _{g}\left(u_{q, i} u_{q, j}\right)^{v}} .
$$

For $S_{k, e}^{v}$ it is possible to construct sector, using the ideas explained in Section 2. For the nonlinearities belonging to class $F_{m}$, it is easy to estimate influence which on her render the elements of vector $U_{n}$ setting structure of function $f(U, n) \in F_{m}$. For this purpose it is possible to take advantage of the approach offered in [21].

\section{Conclusion}

The concept of a field of structures for nonlinear static systems on set of secants is introduced. The mode of construction of a sector condition for a nonlinear part of system is offered. Algorithms and methods of decision-making in the form of nonlinearity are described. For the nonlinearity described by a class polynomial of functions, the adaptive approach for an estimation of her structure in the conditions of uncertainty is offered. The structure on which it is possible to make a solution on nonlinearity of system is introduced.

\section{References}

[1] N. S. Rajbman and V. M. Chadeev, "Building of models of processes of manufacture," Energy, Moscow, 1975.

[2] L. Ljung, "System Identification: Theory for User," Prentice-Hall, Englewood Cliffs, New Jersey, 1987.

[3] E. K. Berndt, B. H. Hall, R. E. Hall, and J. A. Hausman, "Estimation and Inference in Nonlinear Structural Models," Annals of Economic and Social Measurement, Vol. 3, No. 4, 1974, pp. 653-665.

[4] J. Madár, J. Abonyi and F. Szeifert, "Genetic Programming for the Identification of Nonlinear Input-Output Models," Industrial \& Engineering Chemistry Research, Vol. 44, No. 9, 2005, pp. 3178-3186.

[5] B. McKay, M. Willis, D. Searson, and G.Montague, "Non-Linear Continuum Regression Using Genetic Programming," 2005. http://www.staff.ncl.ac.uk/d.p.searson/ docs/NLCR_GP3.pdf

[6] L. Ljung, "System Identification: Nonlinear Models," Berkeley, 2005.

[7] G. R. Liu, "Nonlinear identification and control. A Neural Network Approach,” Springer-Verlag, London, 2001.

[8] M. Norgaard, O. Ravn, N. K. Poulsen and L. K. Hansen, "Neural Networks for Modelling and Control of Dynamic Systems: A Practitioner's Handbook," Springer-Verlag, London, 2001.

[9] G. W. Irwin, K. Warwick and K. J. Hunt, Eds., "Neural Network Applications in Control," The Institution of Electrical Engineers, London, 1995. 
[10] G. Dreyfus, "Neural Networks: Methodology and Applications," Springer-Verlag, Berlin, Heidelberg, 2005.

[11] N. Sundararajan, P. Saratchandran and Y. W. Lu, "Radial Basis Function Neural Networks with Sequential Learning: MRAN and its Applications," World Scientific Publishing Co, Singapore, 1999.

[12] E. Righeto, L. H. M. Grassi and J. A. Pereira, "Nonlinear Plant Identification by Wavelets," ABCM Symposium Series in Mechatronics, Vol. 1, 2004, pp. 392-398.

[13] T. Sato, and M. Sato, "Structural Identification Using Neural Network and Kalman Filter Algorithms," Structural Engineer/Earthquake Engineer, JSCE, Vol. 14, No. 1, 1997, pp. 23s -32s.

[14] S. F. Masri, J. P. Caffrey, T. K. Caughey, A. W Smyth and A. G. Chassiakos, "Direct Identification of the State Equation in Complex Nonlinear Systems," ICTAM04Complex Nonlinear Systems, 2003, pp. 1-2.

[15] L.A. Aguirre, M. F. S. Barroso, R. R. Saldanha and E. M. A. M. Mendes, "Imposing Steady-State Performance on Identified Nonlinear Polynomial Models by Means of Constrained Parameter Estimation," IEEE Proceedings of Control Theory and Applications, Vol. 151, No. 2, 2004, pp. 174-179.

[16] V. M. Chadeev and V. B. Ilyushin, "Identification Technique Concerning a Priori Information on Plant Parameters," Proceedings of the V International Conference "System Identification and Control Problems" SICPRO'06,
Institute of Control Sciences, Moscow, 30 January-2 $\mathrm{Fe}$ bruary 2006, pp. 1091-1105.

[17] S. M. Spottswood, "Identification of Nonlinear Parameters from Experimental Data for Reduced Order Models," University of Cincinnati, Cincinnati, 2006.

[18] M. Espinoza, J.A.K. Suykens and B. De Moor, "Kernel Based Partially Linear Models and Nonlinear Identification," IEEE Transactions on Automatic Control, Vol. 50, No. 10, 2005, pp. 1602-1606.

[19] D. Graupe, "Identification of Systems," Robert E. Krieger Publishing Co., Huntington, New York, 1976.

[20] M. A. Ajzerman, "About One Problem, a Concerning Stability 'in Big' Dynamic Systems," Successes of Mathematical Sciences, Vol. 4, 1949, pp. 186-188.

[21] N. N. Karabutov, "Structural System Identification: Information Structure Analysis," URSS/Izd. Dom "Librokom”, Moscow, 2009.

[22] N. N. Karabutov, "Selection of the Structure of a Model in Processing the Results of Measurements in Control Systems," Measurement Techniques, Vol. 51, No. 9, 2008, pp. 960-966.

[23] N. N. Karabutov, "Structure Field Construction for Nonlinear Static Systems Based on Measurement Data Processing," Measurement Techniques, Vol. 52, No. 12, 2009, pp. 1281-1288. 